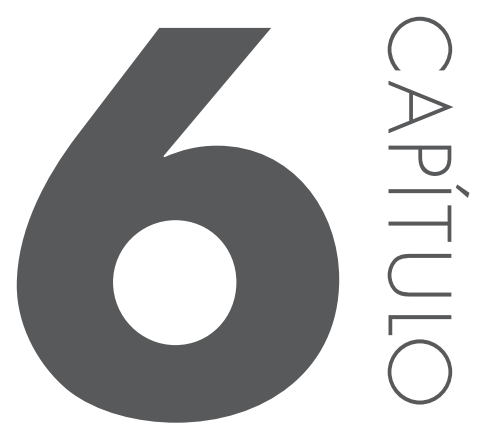

\title{
MODELAGEM MATEMÁTICA DE SISTEMAS MECÂNICOS: ANÁLISE VIBraTÓRIA
}

Gonçalves F., Rosane ${ }^{~ *}$;

Napoleão R., Marcos ${ }^{2}$

1 Universidade Federal de Goiás

2 Universidade Federal de Goiás

* email: rosannymat@hotmail.com 
Resumo: Na modelagem matemática as equações diferenciais incorporam as características importantes do processo real, através da operação de um modelo físico construído. Nas investigações científicas em que não é possível a solução analítica do problema, o modelo matemático numérico é de grande importância na validação do processo, onde são comparadas as previsões com resultados experimentais. Uma das áreas das ciências aplicadas que mais se utilizam da modelagem matemática é a mecânica. Esta área estuda o movimento, dentre suas subdivisões destacase a mecânica dos corpos rígidos (estática e dinâmica, respectivamente corpos que estão em repouso e movimento), a mecânica do contínuo (comportamento dos corpos sujeitos a forças externas) e a mecânica dos fluídos (comportamento físico de líquidos, gases e suas propriedades). Dentro da mecânica do contínuo, existe ainda um tópico conhecido como vibrações mecânicas. Tais fenômenos são indesejáveis, pois comprometem máquinas e estruturas, por isso é desejável que sejam reduzidas ou eliminadas completamente. Neste trabalho estuda-se o comportamento de duas molas acopladas em uma barra móvel, estando esta sujeita à forças externas. Através da discretização das equações do movimento, chega-se a um sistema de equações diferenciais de primeira ordem. Os resultados numéricos foram obtidos através do método de Runge-Kutta de quarta ordem cujo algoritmo fora implementado no software MATLAB. Os resultados numéricos mostram que após uma rápida oscilação $\bigcirc$ movimento das molas tendem à uma linearidade.

\section{Palavras-chave: Modelagem matemática; equações de Euler-Lagrange; vibrações mecânicas}

Gonçalves F., Rosane; Napoleão R., Marcos; "MODELAGEM MATEMÁTICA DE SISTEMAS MECÂNICOS: ANÁLISE VIBRATÓRIA", p. 88-100 . In: Seminário de Pesquisa, Pós-Graduação e Inovação da Regional Catalão (2. : 2014 : Goiás) Coletânea Interdisciplinar em Pesquisa, Pós-Graduação e Inovação - Volume 4 : Ciências Exatas e Tecnológicas. Anais [livro eletrônico] / organizado por Adriana Freitas Neves, Idelvone Mendes Ferreira, Maria Helena de Paula, Petrus Henrique Ribeiro dos Anjos. São Paulo: Blucher, 2015. ISBN: 978-85-8039-115-2, DOI 10.5151/978859788580391152-V4_Cap6 


\section{Introduc̣ão}

Neste trabalho considera-se um modelo matemático numérico, de um sistema acoplado por duas molas, onde se pretende estudar o movimento vibratório do sistema associado. Para a análise do modelo serão consideradas as equações que descrevem o movimento, a velocidade e a aceleração de um corpo.

A solução geral do movimento ondulatório é dada por

$$
\mathrm{x}=\mathrm{x}_{\mathrm{m}} \sin \left(\omega_{\mathrm{n}} \mathrm{t}+\phi\right)
$$




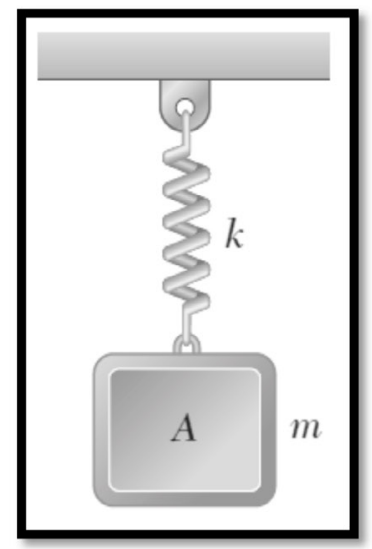

Figura 1 sistema massa mola. [1]

De acordo com a mecânica geral a velocidade e aceleração de um bloco de massa m são dadas por

$$
\begin{gathered}
v=\dot{x}=x_{m} \omega_{n} \cos \left(\omega_{n} t+\phi\right) \\
a=\ddot{x}=-x_{m} \omega_{n}^{2} \sin \left(\omega_{n} t+\phi\right)
\end{gathered}
$$

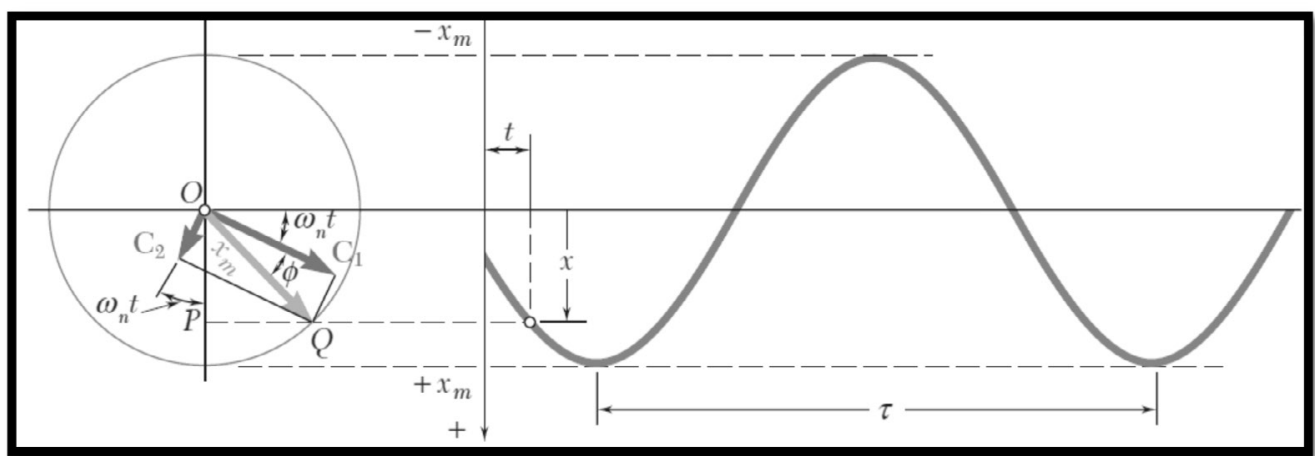

Figura 2 curva seinodal. [1]

A curva senoidal representada na figura 2 acima descreve a trajetória no plano deslocamento-tempo. Segue da periodicidade da função seno que, quando o ângulo $\omega_{\mathrm{n}} \mathrm{t}$ aumenta em um comprimento de $2 \pi$ rad o movimento do bloco $\mathrm{A}$ completa o ciclo, pois $2 \pi$ rad é o intervalo de tempo necessário paa o sistema completar um ciclo inteiro de movimento. $O$ valor representado por $\tau_{n}$, correspondente ao menor tempo $t_{\text {, }}$ necessário para se completar uma volta, e é chamado de período de vibração livre, cuja fórmula é dada por

$$
\tau_{\mathrm{n}}=\frac{2 \pi}{\omega_{\mathrm{n}}}
$$

O número de ciclos por unidade de tempo é conhecido como frequência natural de vibração, cuja fórmula é dada por 


$$
\mathrm{f}_{\mathrm{n}}=\frac{1}{\tau_{\mathrm{n}}}=\frac{\omega_{\mathrm{n}}}{2 \pi}
$$

Segue das equações (2) e (3) que a amplitude máxima da velocidade e aceleração são dadas por

$$
\mathrm{v}_{\mathrm{m}}=\mathrm{x}_{\mathrm{m}} \omega_{\mathrm{n}} \quad \mathrm{a}_{\mathrm{m}}=\mathrm{x}_{\mathrm{m}} \omega_{\mathrm{n}}^{2}
$$

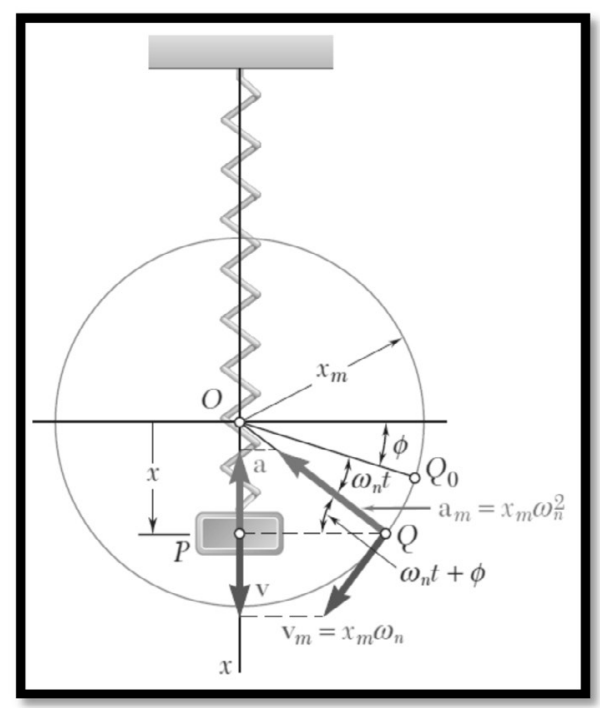

Figura 2 circunferência auxiliar. [1]

\subsection{Vibrações Mecânicas}

Da teoria de vibrações mecânica temos que, corpos sujeitos a forças restauradoras (Lei de Hooke) tendem a descrever movimentos ondulatórios. No caso de sistemas massa \mola, quando um corpo está em equilíbrio estático, são consideradas duas forças, W (a força de seu peso) e T(a força exercida pela mola)

$$
\mathrm{T}=\mathrm{k} \delta_{\mathrm{st}} \quad \mathrm{W}=\mathrm{k} \delta_{\mathrm{st}}
$$

A intensidade da resultante das duas forças exercidas sobre o corpo é

$$
\mathrm{F}=\mathrm{W}-\mathrm{k}\left(\delta_{\mathrm{st}}+\mathrm{x}\right)=-\mathrm{kx}
$$




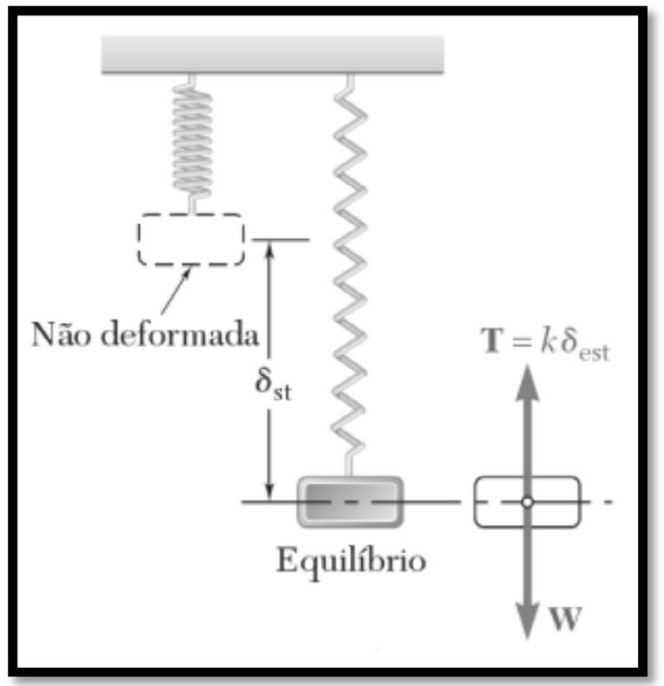

Figura 4 sistema massa-mola. [1]

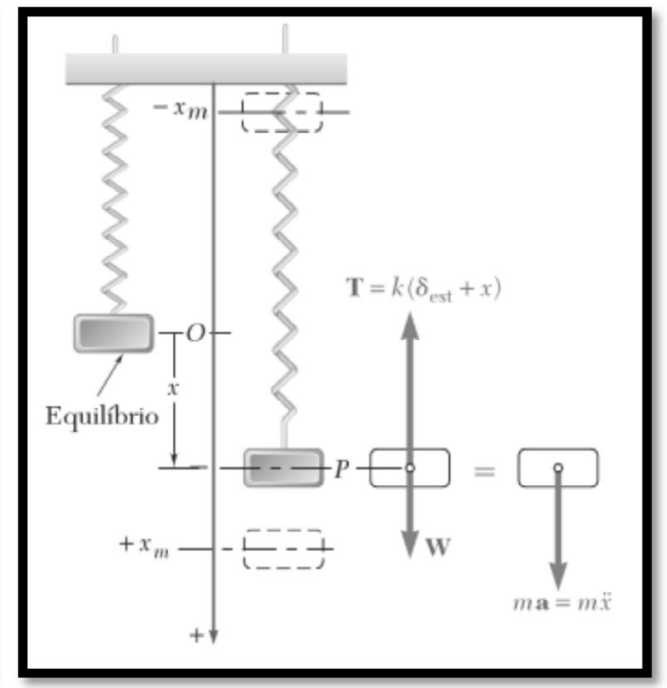

Figura 5 sistema massa-mola. [1]

Substituindo (8) na equação fundamental $\mathrm{F}=\mathrm{ma}$, podemos concluir que aceleração é proporcional ao deslocamento, mantendo a mesma direção, porém, com sentido oposto, obtemos o movimento harmônico simples (segunda lei de Newton) representado por

$$
\mathrm{m} \ddot{\mathrm{x}}+\mathrm{kx}=0
$$

onde, m é a massa do bloco $A, k$ a constante de rigidez da mola, $\ddot{x}$ é a aceleração e $\mathrm{x}$ representa o deslocamento.

\subsection{Movimento sujeito à força peso}

O estudo consiste em um modelo matemático numérico, onde deseja-se saber 0 movimento do sistema nos pontos $B$ e $C$, quando o mesmo está sujeito á força peso. $O$ movimento vibratório acontece quando o sistema é deslocado de sua posição de equilíbrio. Através da força restauradora da mola o sistema passa a oscilar em torno do ponto de equilíbrio.

Quando o sistema volta a posição original, o ciclo de movimento está completo, o intervalo de tempo para que o ciclo se complete chama-se período de vibração. $O$ número de ciclos de movimento por unidade de tempo é a frequência, e o deslocamento máximo do sistema a partir da sua posição de equilíbrio é a amplitude da vibração.

Inicialmente, considere uma barra móvel sujeita a seu próprio peso, 


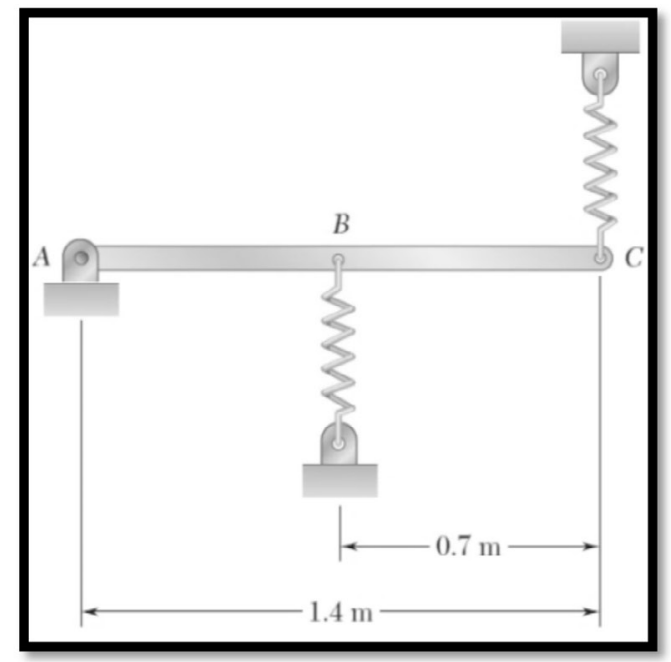

Figura 6 barra móvel.

ela possui três acoplamentos $A, B$ e $C$ e duas molas. Ambas estão acopladas apenas nos acoplamentos B e C. Considerando o ponto C como acoplamento 1 e o ponto B como acoplamento 2, quando uma força peso é aplicada em C têm-se um deslocamento. Aplicando as equações do movimento chega-se a:

$$
\begin{aligned}
& \mathrm{m}_{1} \ddot{y}_{1}-\mathrm{k}_{1} \mathrm{y}_{1}-\mathrm{p}=0 \\
& \mathrm{~m}_{2} \ddot{y}_{2}+\mathrm{k}_{2} \mathrm{y}_{2}-\operatorname{sen}\left(\tan ^{-1}\left(\frac{\mathrm{y}_{1}}{0.7}\right)\right) \mathrm{p}=0
\end{aligned}
$$

Transformando as equações (9) e (10) em sistema de ordem um, tem-se

- em relação à $y_{1}$,

$$
\mathrm{y}_{11}=\mathrm{y}_{1}, \quad \mathrm{y}_{12}=\dot{\mathrm{y}}_{1}, \quad \dot{\mathrm{y}}_{11}=\dot{\mathrm{y}}_{1}=\mathrm{y}_{12}, \quad \dot{\mathrm{y}}_{12}=\ddot{\mathrm{y}}_{1} \text {. }
$$

- em relação à $\mathrm{y}_{2}$,

$$
\mathrm{y}_{21}=\mathrm{y}_{2}, \quad \mathrm{y}_{22}=\dot{\mathrm{y}}_{2}, \quad \dot{\mathrm{y}}_{21}=\dot{\mathrm{y}}_{2}=\mathrm{y}_{22}, \quad \dot{\mathrm{y}}_{22}=\ddot{\mathrm{y}}_{2} \text {. }
$$

Substituindo esses valores em (9) tem-se:

Disto

$$
\dot{\mathrm{y}}_{12}=\frac{\mathrm{k}_{1}}{\mathrm{~m}_{1}} \mathrm{y}_{11}+\frac{\mathrm{p}}{\mathrm{m}_{1}} \text {. }
$$

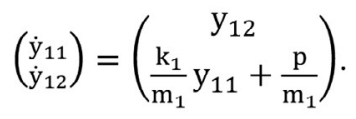


Substituindo esses valores em (10) tem-se:

$$
\dot{\mathrm{y}}_{22}=-\frac{\mathrm{k}_{2}}{\mathrm{~m}_{2}} \mathrm{y}_{21}+\frac{1}{\mathrm{~m}_{2}} \operatorname{sen}\left(\tan ^{-1}\left(\frac{\mathrm{y}_{11}}{0.7}\right)\right) \mathrm{p} .
$$

Disto

$$
\left(\begin{array}{l}
\dot{y}_{21} \\
\dot{y}_{22}
\end{array}\right)=\left(\begin{array}{c}
\mathrm{y}_{22} \\
-\frac{\mathrm{k}_{2}}{\mathrm{~m}_{2}} \mathrm{y}_{21}+\frac{1}{\mathrm{~m}_{2}} \operatorname{sen}\left(\tan ^{-1}\left(\frac{\mathrm{y}_{11}}{0.7}\right)\right) \mathrm{p}
\end{array}\right)
$$

Então de (11) e (12) tem-se o sistema de equações

$$
\left(\begin{array}{c}
\dot{\mathrm{y}}_{11} \\
\dot{\mathrm{y}}_{12} \\
\dot{\mathrm{y}}_{21} \\
\dot{\mathrm{y}}_{22}
\end{array}\right)=\left(\begin{array}{c}
\mathrm{y}_{12} \\
\frac{\mathrm{k}_{1}}{\mathrm{~m}_{1}} \mathrm{y}_{11}+\frac{\mathrm{p}}{\mathrm{m}_{1}} \\
\mathrm{y}_{22} \\
-\frac{\mathrm{k}_{2}}{\mathrm{~m}_{2}} \mathrm{y}_{21}+\frac{1}{\mathrm{~m}_{2}} \operatorname{sen}\left(\tan ^{-1}\left(\frac{\mathrm{y}_{11}}{0.7}\right)\right) \mathrm{p}
\end{array}\right) .
$$

onde $\dot{y}_{11}$ é a posição da mola $1, \dot{y}_{12}$ é a velocidade da mola $1, \dot{y}_{21}$ é a posição da mola 1 e $\dot{y}_{22}$ é a velocidade da mola 2.

Para resolver esse problema computacionalmente, foi usado o método de RungeKutta(RK) de quarta ordem, cujo o algoritmo fora implementado no MATLAB. No algoritmo as variáveis e as constantes do sistema ficam $\dot{\mathrm{y}}_{11}=\mathrm{y}(2)$ posição da mola 1, $\dot{\mathrm{y}}_{12}=\mathrm{y}(1)$ velocidade da mola 1, $\dot{\mathrm{y}}_{21}=\mathrm{y}(3)$ posição da mola 2 e $\dot{\mathrm{y}}_{22}=\mathrm{y}(4)$ velocidade da mola 2 . $\mathrm{E}_{1}=0.1$ para a mola $1, \mathrm{~m}_{1}=1.1$ massa da mola $1, \mathrm{p}=9.81$ força peso, $\mathrm{k}_{2}=0.2$ para a mola 2 e $\mathrm{m}_{2}=1.2$ massa da mola 2 .

\subsection{Resultados Numéricos}

Os gráficos abaixo são os resultados numéricos obtidos computacionalmente, através do método de Runge-Kutta de quarta ordem implementado no MATLAB. Eles representam o ciclo de movimento completo do sistema nos pontos $B$ e $C$, quando o mesmo está sujeito á força peso. 


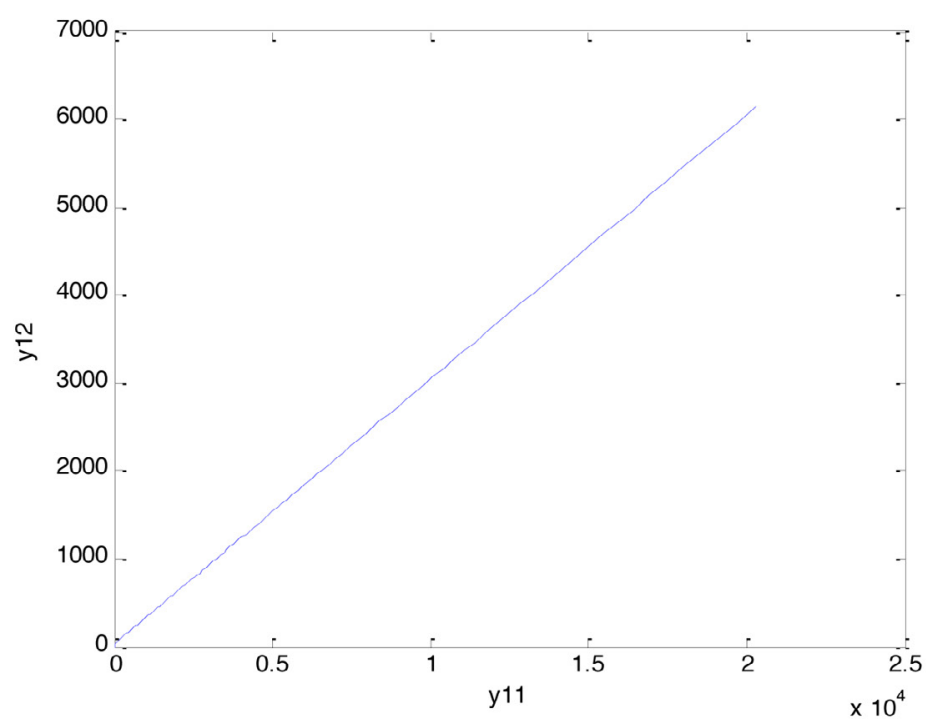

Figura 1 posição versus velocidade da mola 1.

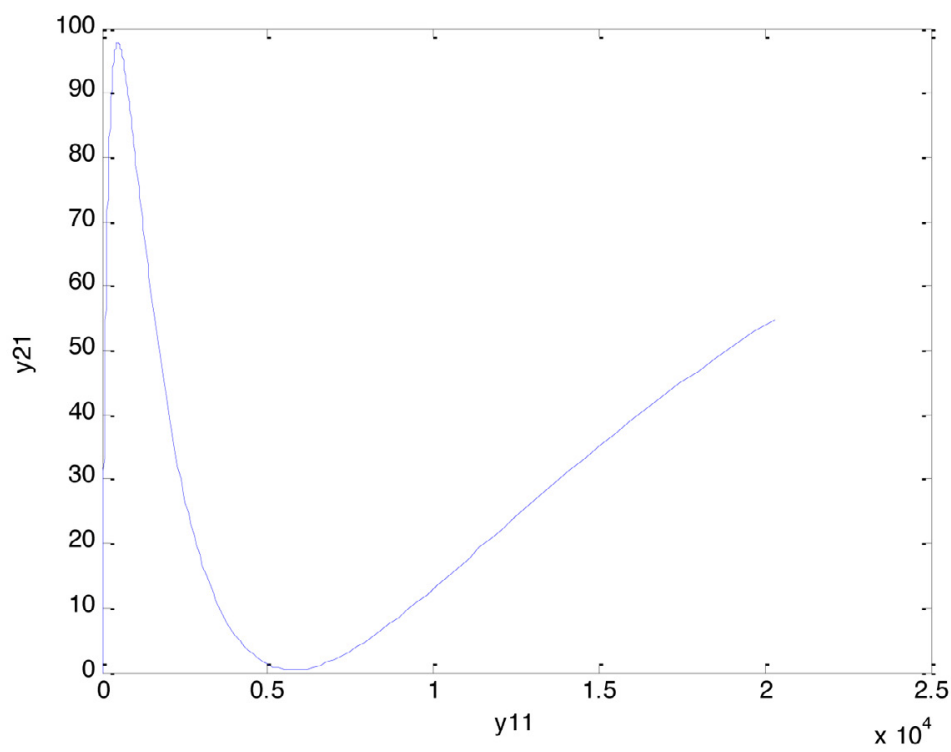

Figura 2 posição da mola 1 versus posição da mola 2 . 


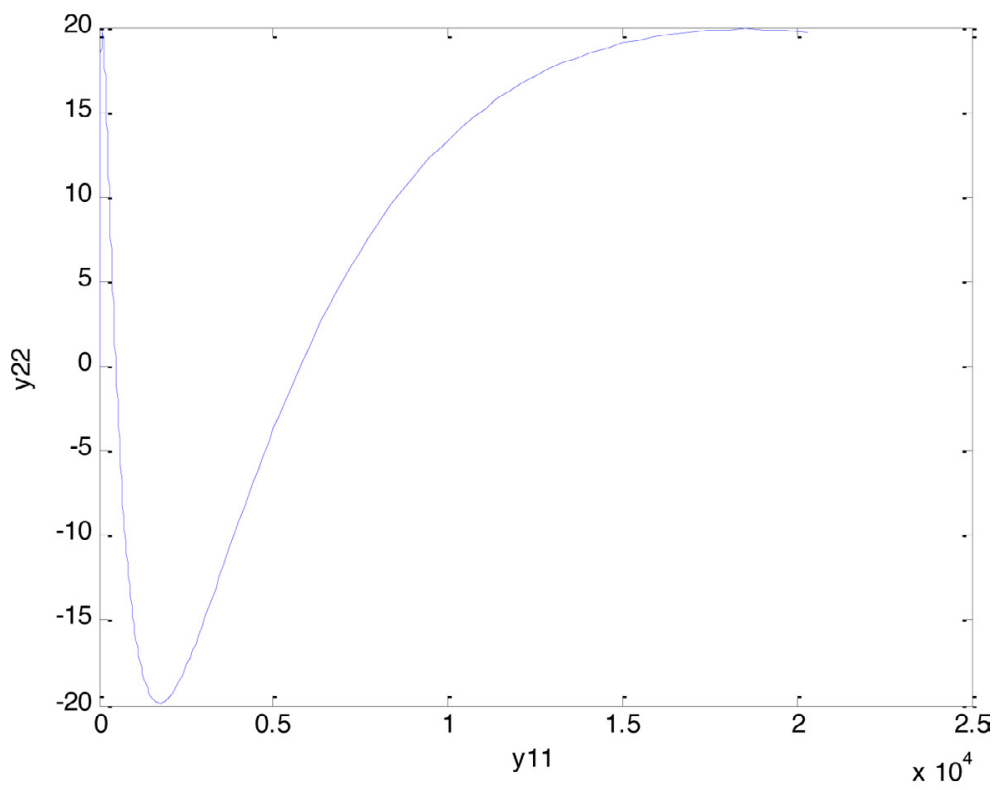

Figura 3 posição da molal versus velocidade da mola 2 .

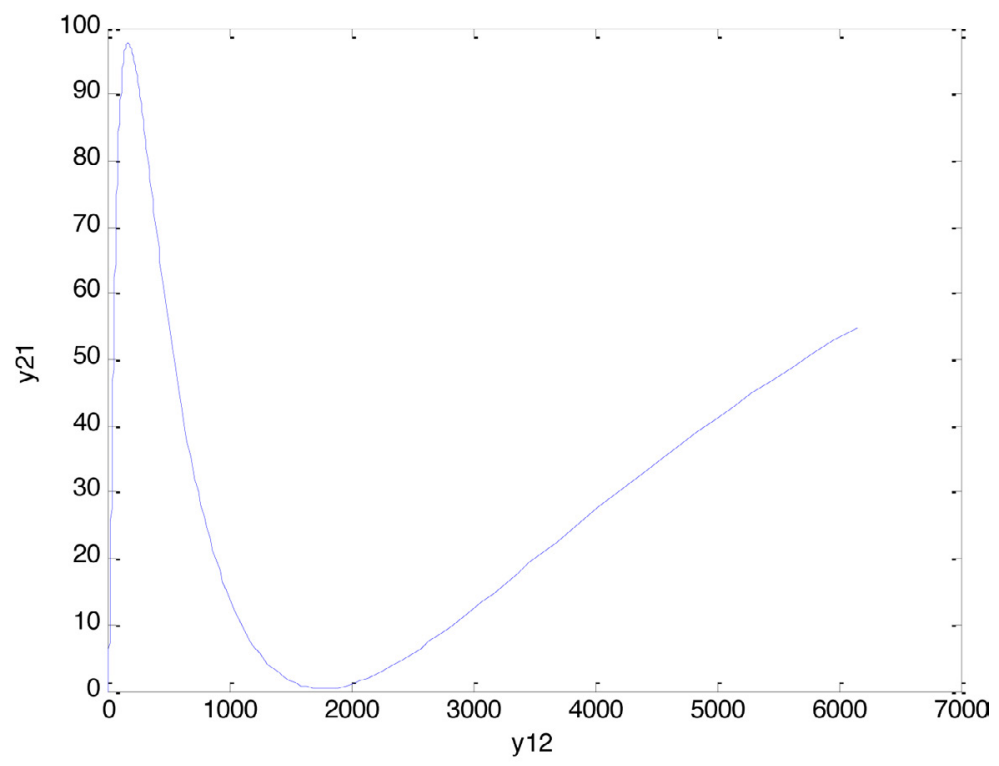

Figura 4 velocidade da molal versus posição da mola 2. 


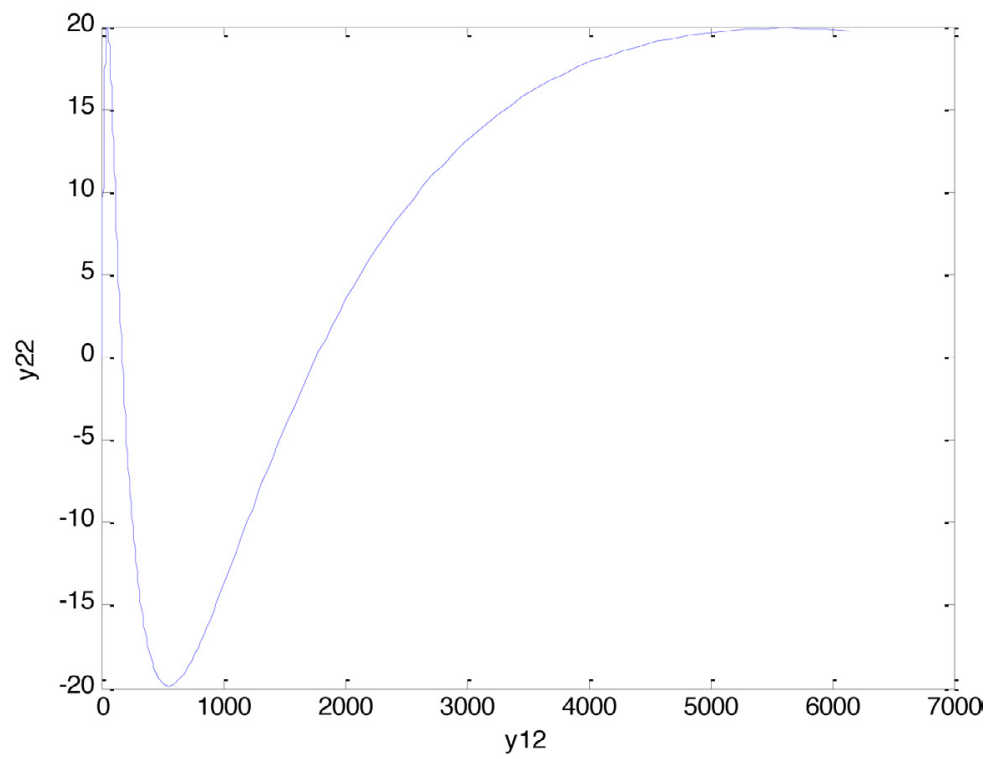

Figura 5 velocidade da mola 1 versus velocidade da mola 2 .

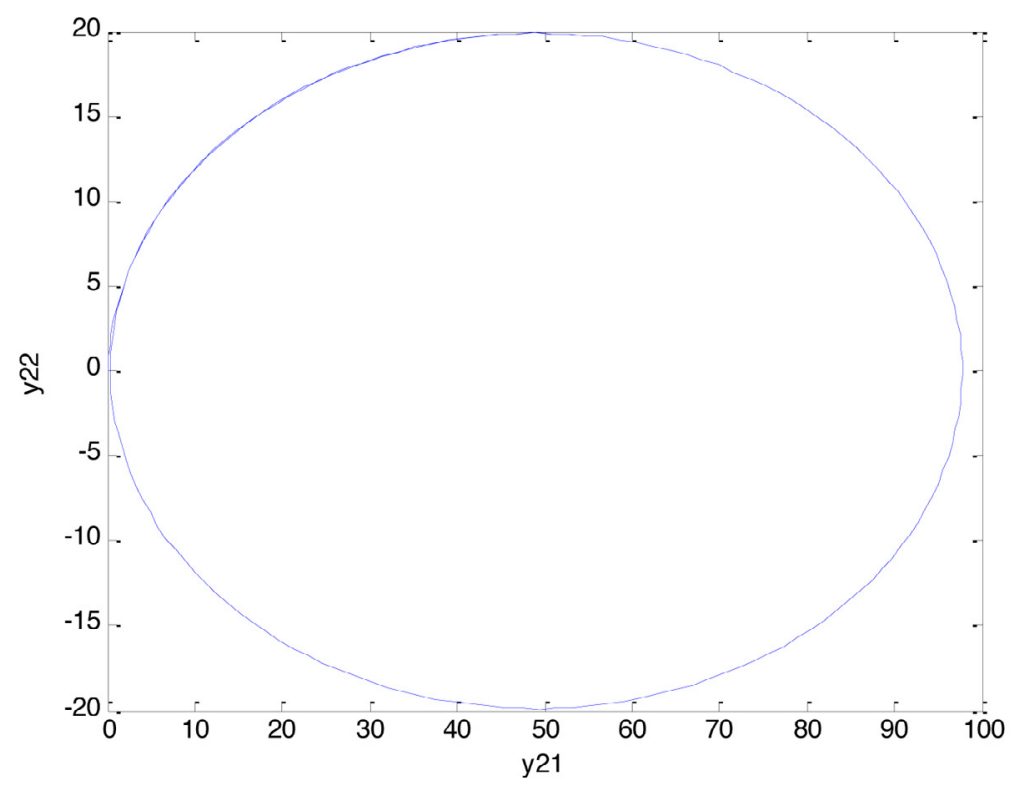

6 posição versus velocidade da mola 2 . 


\subsection{Análise do processo}

Analisando o movimento das molas, percebe-se que em relação a posição e a velocidade da mola 1 , elas tendem a manter uma aproximação linear. Em relação a posição da mola 1 e a posição da mola 2, a mola 2 acompanha a mola 1 rápido, decresce rápido e entram em equilíbrio. Em relação a posição da mola 1 e a velocidade da mola 2, a resposta da mola 1 é rápida para a mola 2, decresce rápido e entram em equilíbrio. Em relação a velocidade da mola 1 e a posição da mola 2, velocidade da mola 1 age com uma força, a mola 2 responde rápido e passa a ter um equilíbrio linear. Em relação a velocidade da mola 1 e da mola 2, a velocidade da mola 2 tem resposta rápida à mola $1 \mathrm{em}$ um intervalo curto, e tendem a chegar a uma posição de equilíbrio. Em relação a posição e a velocidade da mola 2, elas passam a ter um equilíbrio periódico, ou seja, passam a ter um movimento oscilante, uma com relação a outra.

\subsection{Conclusão}

Observa-se que este é um modelo numérico simples, servindo de base para resolução de problemas mais complexos, que serão tratados no decorrer do mestrado.

Após um determinado instante de tempo o sistema atinge sua posição de equilíbrio. Assim, o sistema após excitação externa tende a se manter em movimento oscilatório ao redor de sua posição de equilíbrio, de forma a restaurar sua configuração original. Isto ocorre devido ao atrito, que funciona como uma força de dissipação fazendo o movimento equilibrar. Esse processo é obtido nos resultados numéricos representados nos gráficos de 1 a 6 . 


\section{Mathematical Modelling of Mechanical Systems: Vibration Analysis}

Abstract: In mathematical modeling differential equations incorporate the important features of the actual process, through the operation of a physical model constructed. In scientific investigations it is not possible analytical solution of the problem, the numerical mathematical model is of great importance in the validation process, where the predictions are compared with experimental results. Mechanics is a branch of applied sciences in which the mathematical tools is more used. This area study the movement of particles and bodies, among its subdivisions highlights the mechanics of rigid bodies (static and dynamic, respectively bodies that are at rest and movement), the continuum mechanics (the behavior of bodies subjected to external forces) and fluid mechanics (physical behavior of liquids, gases and their properties). In continuum mechanics, there is still a topic known to mechanical vibrations. Such phenomena are undesirable because compromise machines and structures, so it is desirable to be reduced or eliminated completely. Here we study the behavior of two coupled springs in a mobile bar, when it is subjected to external forces. Through the discretization of the equations of motion, we arrive at a system of differential equations of first order for problem resolution. The numerical results were obtained using the Runge-Kutta fourth order algorithm which was implemented in MATLAB. Numerical results show that after a rapid oscillation movement of the springs tend to linearity.

Keywords: Mathematical modeling; Euler-Lagrange equations; mechanical vibrations

\section{Referências bibliográficas}

BEER, Ferdinand P.; JOHNSTON, Russell E.; MAZUREK, David F.; CORNWELL, Phillip J.; EISENBERG, Elliot R. Vector mechanics for engineers: statics and dynamics, McGraw-Hill. New York: 2010. 